\title{
Lymphosarcoma Involving Spleen
}

National Cancer Institute

\section{Source}

National Cancer Institute. Lymphosarcoma Involving Spleen. NCI Thesaurus. Code C26960.

An antiquated term that refers to a non-Hodgkin lymphoma composed of small and medium sized lymphocytes affecting the spleen. 